\section{Journal homepage: http://www.journalijar.com Journal DOI:10.21474/IJAR01}

RESEARCH ARTICLE
INTERNATIONAL JOURNAL

OF ADVANCED RESEARCH

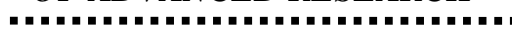

\title{
Prescribing Errors in Prescription Orders Containing Non-Steroidal Anti-Inflammatory Drugs: A Case Study of District Khairpur, Sindh, Pakistan.
}

\author{
Abdul Sami Shaikh ${ }^{1,2}$, Wenlong $\mathrm{Li}^{1}$, Chunmin Wei ${ }^{1}$, Meimei Gao ${ }^{1}$, Chunmei Geng ${ }^{1}$, Lei Shi ${ }^{1}$ and ${ }^{*}$ Ruichen \\ Guo'. \\ 1. Institute of Clinical Pharmacology, Qilu Hospital of Shandong University, Jinan-250012, China. \\ 2. Department of Pharmacy, Shah Abdul Latif University, Khairpur, Sindh, Pakistan.
}

\section{Manuscript Info}

\section{Manuscript History:}

Received: 18 March 2016

Final Accepted: 16 April 2016

Published Online: May 2016

Key words:

Khairpur, NSAIDs, Medical errors,

Outpatients Prescriptions,

Prescribing errors.

*Corresponding Author

Ruichen Guo

\begin{abstract}
A prescription error is a failure in the prescription writing process which results in a wrong instruction about one or more of the normal features of a prescription. The objective of this study is to identify the extent of prescribing errors in prescriptions containing Non-steroidal antiinflammatory drugs (NSAIDs) and to find appropriate solutions for reducing these errors because NSAIDs are one of the widely misused drugs. A total of 479 prescriptions containing NSAIDs were collected from various outpatient clinical settings of district Khairpur Pakistan and analyzed retrospectively to identify the common prescribing errors, i.e. omission errors and commission errors as per prescription writing guidelines/parameters established by the World Health Organization and authenticated drug references, such as, The Drug information book and the British National Formulary (BNF). Only $21(4.4 \%)$ prescriptions were found error free and the remaining $458(95.6 \%)$ prescriptions contained different types of errors. Errors were divided into omission and commission errors. Among omission errors, most of the prescriptions were missing key information related to the patient, such as patient diagnosis, which was not written in $84 \%$ of prescriptions. Among information pertaining to the prescriber, the registration number was missing in $88.9 \%$ of prescriptions. Among drug related information, duration of therapy was not written in $82.8 \%$ of prescriptions. Among commission errors, $85.2 \%$ of prescriptions were ambiguously written. A significant percentage of omission and commission errors were found in routine practice. It is strongly recommended that computerized physician order entry (CPOE) and continuous educational training programs for prescribers to be implemented in order to reduce vital prescribing errors and prescriptions should be reviewed by pharmacists in order to reduce the extent of these serious and fatal errors.
\end{abstract}

Copy Right, IJAR, 2016,. All rights reserved.

\section{Introduction:-}

Medication errors are among the utmost frequent incidents described in hospitals. Prescribing errors are potentially the most grave of all medication errors ${ }^{[1]}$. Prescriptions are written requests for drugs or medications prescribed by legally qualified prescribers. Drugs are mainly classified into two legal categories, i.e. prescription drugs or legend drugs and non-prescription drugs or (over the counter drug) OTC drugs ${ }^{[2]}$. Prescriptions are very vital because they become a medico-legal document once they are signed by the legal prescribing authority, and thus they are mandatory to be written completely, legibly and also free of error ${ }^{[3]}$. The legal requirements for writing a standard prescription differ from region to region. Generally a prescription includes (a) name, qualification, contact number, address, registration number and signature of the prescriber; (b) name, gender, age, weight and diagnosis of the 
patient; (c) brand or generic name of drugs, dosage form, strength, frequency, dose, duration of therapy, quantity of drug to be dispensed and (d) date, directions for use and specific cautions required to be taken for use ${ }^{3,4]}$. The prescriber is not always a doctor but can also be paramedical staff, such as a medical assistant, a technician, a midwife or a nurse. The dispenser is not always a pharmacist, but can be a pharmacy technician, a pharmacy assistant or a nurse. Every country has its own and different standards for the minimum information required to include in prescription and its own laws and regulations to define which drugs require a prescription and who is eligible to write it. Medication errors are the errors resulting in inappropriate use or harm while the medication is in the control of health care professionals and patients ${ }^{[5]}$. Medication errors may be committed by both experienced and inexperienced professionals including: pharmacists, doctors, nurses, supportive personnel (pharmacy technicians), students, clerical personnel (ward clerks), administrators, drug manufacturers, patients and their caregivers and others. The occurrence of medication errors is unknown; valid comparisons of different studies on medication errors are extremely difficult because of differences in variables, measurements, populations and methods ${ }^{[6]}$.Prescribing error is an error which occurs when, as a result of a prescribing decision or prescription writing process, there is an unintentional noteworthy a) decrease in the probability of timely and effective treatment or b) increase the probability of harm ${ }^{[7]}$. Prescribing errors can be classified into two main groups: a) omission errors, which includes those errors in which there is incomplete or missing information in the prescription as well as prescriptions which are unreadable and prescriptions that do not conform to legal requirements; b) commission errors, which includes incorrect or wrongly written information in the prescription ${ }^{[8-9]}$.

The Hand-written prescription process is ineffective, costly and resource-intensive. This method has numerous other limitations such as high proportion of human errors in operating data, and documentation errors which are inevitable ${ }^{[10]}$. A study was done in a hospital of New York State; the authors stated that about 402 common types of dosage form prescribing errors were mainly related to cardiovascular drugs. The factors related to errors of these dosage forms include: Insufficient caregivers and lack of knowledge of the patient, puzzling and inconsistent nomenclature, ignorance of the safety in medicine preparation and packaging design, product marketing and insufficient health care system processes to safeguard patients ${ }^{[11]}$. Prescription errors may lead to serious morbidities and mortalities. In another study of a teaching hospital, it was found that $23.7 \%$ of the patients were affected due to the prescribing of contraindicated drugs and adversely interacting drugs and in total $1.9 \%$ of prescriptions were potentially hazardous ${ }^{[12]}$. Ridley et al. conducted a study on prescribing errors in 24 critical care units of United Kingdom. In that study errors were found in 3,141 (15\%) of the prescriptions in a total of 21,589 prescriptions; among those 3,141 errors, $916(19.6 \%)$ were stated as potentially life threatening errors ${ }^{[13]}$. Furthermore, prescription errors may also lead to an overall increase in cost of the therapy. In February 2000, Dr. Patricia and co-workers evaluated 3,540 prescription orders during a period of one week of interventions done by pharmacy staff. They observed that 351 (9.9\%) prescriptions contained prescribing errors ${ }^{[14]}$. In another study, investigators compared the risk of cardiovascular diseases occurring in users and non-users of NSAIDs, concentrating on three commonly used drugs: Diclofenac, Naproxen and Piroxicam. They observed that there is consistent increased risk of cardiovascular diseases in users of NSAIDs over non-users and all three drugs had a higher risk of cardiovascular events ${ }^{[15]}$. Another study published in September 2001 revealed that there is a 4.1 times increase in the risk of developing upper gastrointestinal problems if the NSAIDs are not used properly ${ }^{[16]}$. A similar type of study was also conducted by Joseph Thomas and his teammates; they stated that patients who were using NSAIDs developed gastrointestinal side effects twice as often $(19.6 \%)$ when compared to a control group $(9.5 \%)^{[17]}$.

NSAIDs are causing serious cardiovascular and gastrointestinal problems which can be decreased with proper and rational use. Several studies regarding prescribing errors have been reported worldwide but unfortunately, sufficient literature is not available on prescribing errors in Pakistan. The aim of this study is to identify the prescribing errors in prescriptions containing Non-steroidal anti-inflammatory drugs (NSAIDs).

\section{Materials and methods:-}

A total of 479 prescriptions containing NSAIDs were collected in district Khairpur and analyzed retrospectively for identifying errors as per standard prescription writing guidelines/parameters provided by World Health Organization ${ }^{[3]}$, authenticated drug references .i.e., Drug information book ${ }^{[8]}$ and British National Formulary (BNF) ${ }^{[19]}$. All prescriptions were collected and then analyzed by registered pharmacist for two main error categories, i.e. Omission Errors and Commission Errors. Among omission errors, important information related to the patient i.e., name, weight, diagnosis etc., and information related to prescriber such as; name, qualification, signature etc., and information related to drug i.e., dose, dosage form, strength etc. was analyzed. Among commission errors, information related to incorrect dose, incorrect strength, incorrect frequency etc. were also identified. Furthermore in our study, information such as brand name, strength, dose, dosage form, frequency, direction of use, was only 
limited to NSAIDs, since NSAIDs are misused widely ${ }^{[16]}$. Prescriptions for drug-drug interactions were evaluated by using the Micromedex.2.0. Drug-Reax database ${ }^{[20]}$.

\section{Data analysis:-}

Data was recorded and analyzed by registered pharmacist in Microsoft office and SPS, and results of these prescribing errors are stated in number and percentages.

\section{Results:-}

A total of 479 prescriptions containing NSAIDs were collected and analyzed for prescribing errors. All 479 prescriptions were evaluated for the existence of vital elements to be present in the manually written prescription orders. While writing the patient prescriptions it is important to include all parameters. Only 21 (4.4\%) prescriptions were found error free and the remaining 458 (95.6\%) prescriptions contained different types of errors and 458 prescriptions containg errors were further evaluated in detail. In the present study, we found that the patient diagnosis was not mentioned in $84.1 \%$ of prescriptions. Patient weight, patient age and patient sex were not written in $83.2 \%, 60 \%$ and $52.4 \%$ of prescriptions respectively. The name of the patient was mentioned in the majority of prescriptions $97.4 \%$. (Table1 and Figure1)

The prescriber's information is also one of the main elements to be mentioned in standard prescriptions. Noncompliance of mentioning prescriber's information was quite high, for example the registration number, qualification, name, telephone number and address was missing in $88.9 \%, 87.1 \%, 53.9 \%, 41.3 \%$ and $34.3 \%$ of prescriptions respectively (Table2 and Figure2). While in $89.7 \%$ prescriptions, the signature of the prescriber was present.

Drug information is an important part of the prescription. In the present study, large numbers of deficiencies regarding drug information were found in the majority of prescriptions: duration of therapy was not mentioned in $82.8 \%$ of prescriptions and other important information such as direction of use, strength, frequency and dose was not mentioned in $78.4 \%, 59 \%, 25.5 \%$ and $24.9 \%$ prescriptions respectively (Table3 and Figure 3 ). In most of the prescriptions, Brand names and dosage forms of drugs were mentioned in $89.7 \%$ and $86.7 \%$, respectively.

Generally, commission errors are vital and may be life threatening to the health of the patient if overlooked. A high percentage of prescriptions, 85.2\%, were illegible or ambiguous. Prescriptions mentioning, dose, dosage form, strength of drugs, duration of therapy and frequency of drug use were further evaluated for commission errors. Ambiguous or nonstandard abbreviations of drug names were mentioned in $40 \%$ prescriptions. In $14.4 \%$ of prescriptions, dose was incorrect and dosage form, strength, duration of therapy, spellings of drug names and frequency of drugs was incorrect in $19.4 \%, 10 \%, 9.6 \%, 12.2 \%$ and $1.9 \%$ prescriptions respectively. Drug Interactions of NSAIDs with other drugs in prescriptions were found in $20.1 \%$ prescriptions. (Table4 and Figure4).

\section{Discussion:-}

In the present study, a total of 479 prescriptions of NSAIDs drugs were collected and assessed for the identification of errors and surprisingly it was observed that the vast majority of prescriptions contained errors (95.6\%) and only $(4.4 \%)$ of prescriptions contained no errors. This prevalence rate of error free prescriptions agrees with Shumaila et al ${ }^{[21]}$ who reported that only $4.5 \%$ of prescriptions contained no errors. Errors were divided into two main categories: omission errors and commission errors. Among omission errors, essential information related to the patient, the prescriber and the drug that had been written or not written was analyzed and it was found that most of

the prescriptions were missing key information. In patient related information, our results regarding missing patient diagnosis, $84.1 \%$ are on par with Shumaila et al. who reported that in $86 \%$ of prescriptions, the diagnosis was absent. Our findings were a little higher than those obtained in studies by Nesar et al ${ }^{[22]}$ and Ghoto et al ${ }^{[23]}$, they found that $75.9 \%$ and $69.58 \%$ of prescriptions respectively. In our study, patient weight was not mentioned in $83.2 \%$

of prescriptions. Shumaila et al and Mugoyela V et al ${ }^{[9]}$ also reported that in $83.9 \%$ and $93.8 \%$ prescriptions respectively, the weight of the patient was not mentioned. The same findings also agreed with Vaishali et al ${ }^{[24]}$ and Irshaid et al ${ }^{[25]}$. While a large number of errors pertaining to age and sex were found, we found that $60 \%$ and $52.4 \%$ of prescriptions respectively were missing these important elements since some drugs are contraindicated in specific genders and some specific age groups. Our results corresponds to Nesar et al who reported that $57.2 \%$ and $61.3 \%$ prescriptions were missing the age and sex, while Vaishali et al reported $11 \%$ and $10 \%$ and Ghoto et al reported 
$25.17 \%$ and $44.05 \%$ prescriptions respectively. The name of the patient was mentioned in $97.4 \%$ of prescriptions in our study and Nesar et al, Vaishali et al and Irshaid et al also corresponds with our findings this, they found that $96.5 \%, 97 \%$ and $94.6 \%$ of prescriptions respectively contained the name of patient.

Most of the prescriptions were missing the basic information of the prescriber. Our results indicate that the prevalence of missing registration number (88.9\%) and qualification $(87.1 \%)$ of prescribers in prescriptions are on par with Shumaila et al, who also reported that $94.5 \%$ and $93.7 \%$ of prescriptions were also missing the same information. Our results of missing information about telephone number (41.3\%) and name of prescriber (53.9\%) are in contrast to $76.5 \%$ and $68.7 \%$ reported by Shumaila et al., while in $10.3 \%$ of prescriptions, signature of prescribers was not mentioned, here our results concur with Shumaila et al, they reported that $11.3 \%$ of prescriptions contain no signature.

Drug information is a key component of medical prescription, it is mandatory to properly indicate this in prescriptions; otherwise life threatening adverse effects can occur. In $82.8 \%$ of prescriptions, duration of therapy or quantity of drug was not mentioned. Usage of NSAIDs for a longer duration can cause serious gastric irritation and drug induced injuries. Banks et al ${ }^{[26]}$ reported that hepatotoxicity was apparent in $24 \%$ of patients by one month, in $63 \%$ of patients by 3 months and in $85 \%$ of patients by 6 months after starting using Diclofenac. Patients need proper directions for using NSAIDs, since these drugs are harmful if used on an empty stomach. These harmful consequences can be avoided by administrating these drugs with food. $78.4 \%$ of prescriptions were missing the information regarding direction for use of NSAIDs and similar results (74.9\%) are reported by Shumaila et al. Likewise, Vaishali reported this error is lower (45.9\%) than ours. Strength of NSAIDs was missing in 59\% of prescriptions, which corresponds to results reported by Shumaila et al (67.3\%) and contrasts to results (3.6\%) reported by Stubbs et al ${ }^{[27]}$, since many strengths of the same brand name or generic name are available in market

i.e., Brufen $200 \mathrm{mg}, 400 \mathrm{mg}, 600 \mathrm{mg}$. Dose and frequency determines efficacy of drug, which was also surprisingly missing in $24.9 \%$ and $25.5 \%$ prescriptions respectively.

Among Commissions errors which are life threating, it was found that $85.2 \%$ prescriptions were written ambiguously, which may result in fatal and serious problems; Shumaila et al reported this error in $77.7 \%$ of prescriptions. Ambiguous or nonstandard abbreviations of drug names were also high; we found this error in $40 \%$ of prescriptions, which can result in very serious and fatal adverse effects. Our findings are similar with Shumaila et al, they found $37.6 \%$. Incorrect dose may lead to failure of drug treatment i.e., under dosage may not produce desired effects and over dosage may cause serious toxic effects. In our study we found that $14.4 \%$ prescriptions had incorrect doses mentioned and incorrect dosage form was also quite high in $19.4 \%$ of prescriptions. Correct frequency of drug use is also very important in maintaining therapeutic concentration of drug in plasma; the correct frequency was mentioned in majority $98.1 \%$ of prescriptions. Mentioning of incorrect, strength (10\%), duration of therapy (9.6\%), spellings (12.2\%) and dosage form (19.4\%) was also high enough and noticeable. Drug-drug interactions were found in $20.1 \%$ of prescriptions and regarding this error, the same results $(19.5 \%)$ were reported by Shumaila et al. During writing prescription of drugs, the prescriber must be careful about drug-drug interactions, if they are not properly considered, these may result in not only reduced therapeutic outcomes but may also result in death of the patient. 


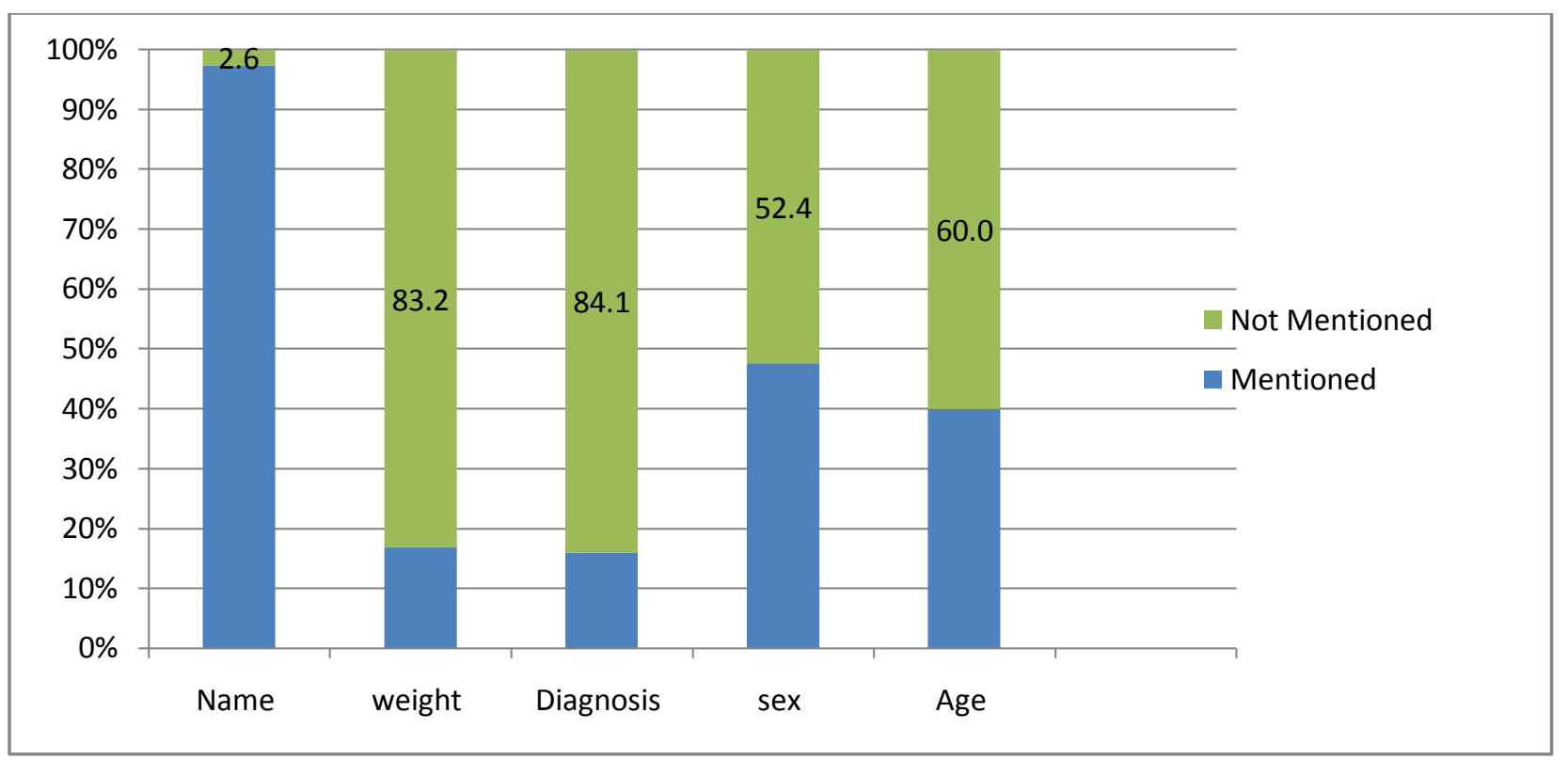

Fig. 1: Graphic presentation of parameters related with patientinformation in prescriptions

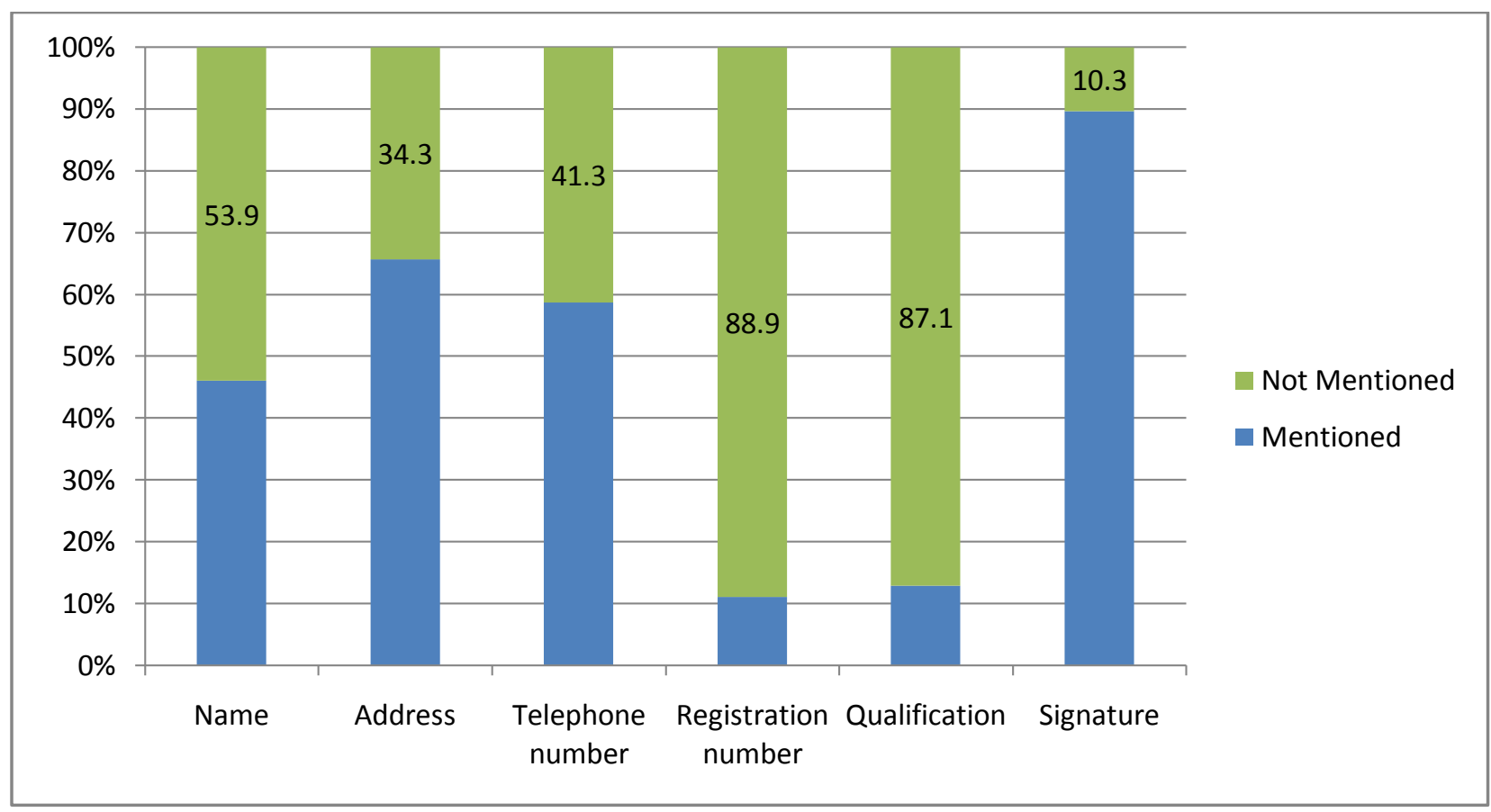

Fig. 2: Graphic presentation of parameters related withprescriber information in prescriptions 


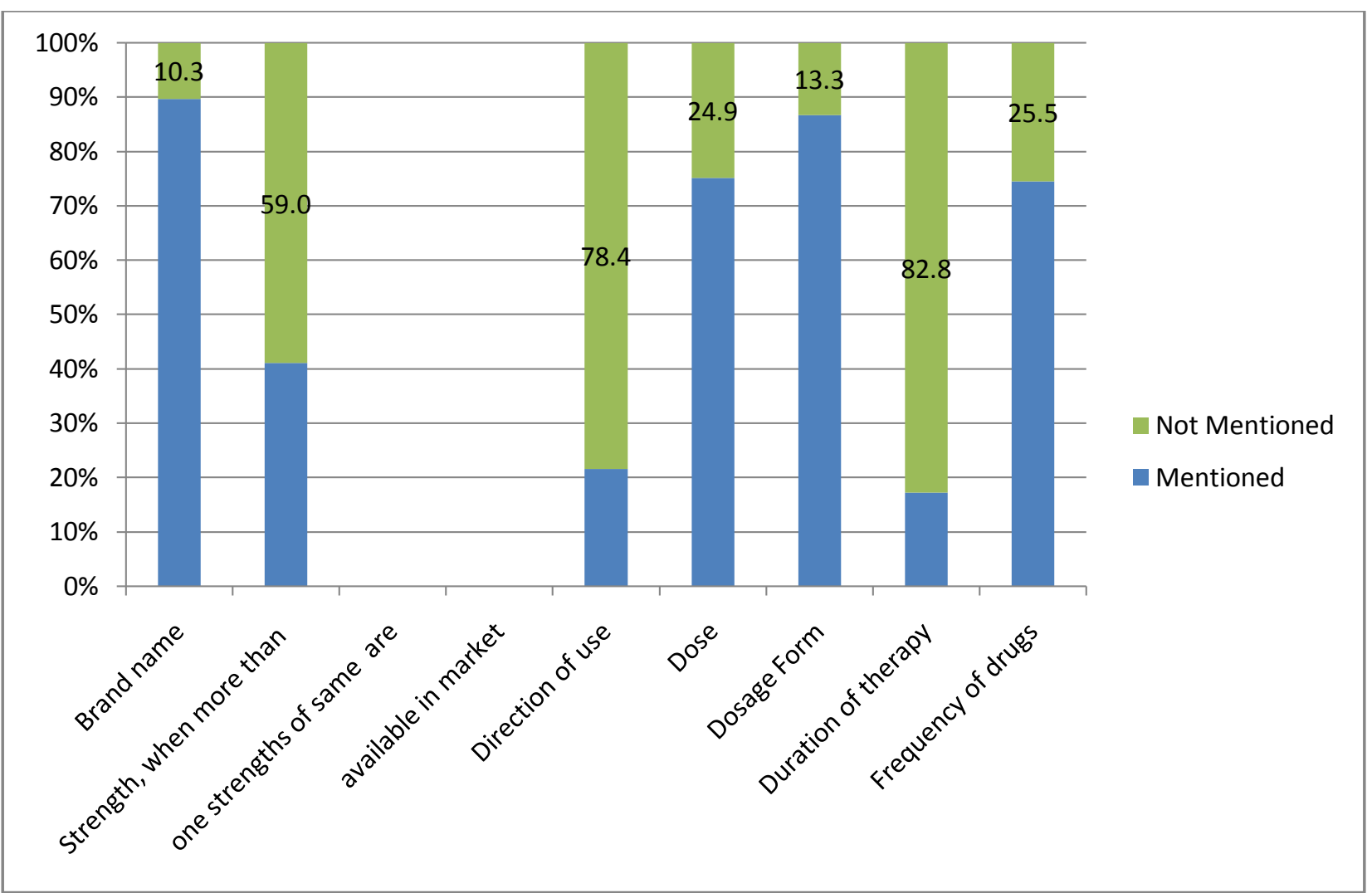

Fig. 3: Graphic presentation of parameters related withdrug information in prescriptions

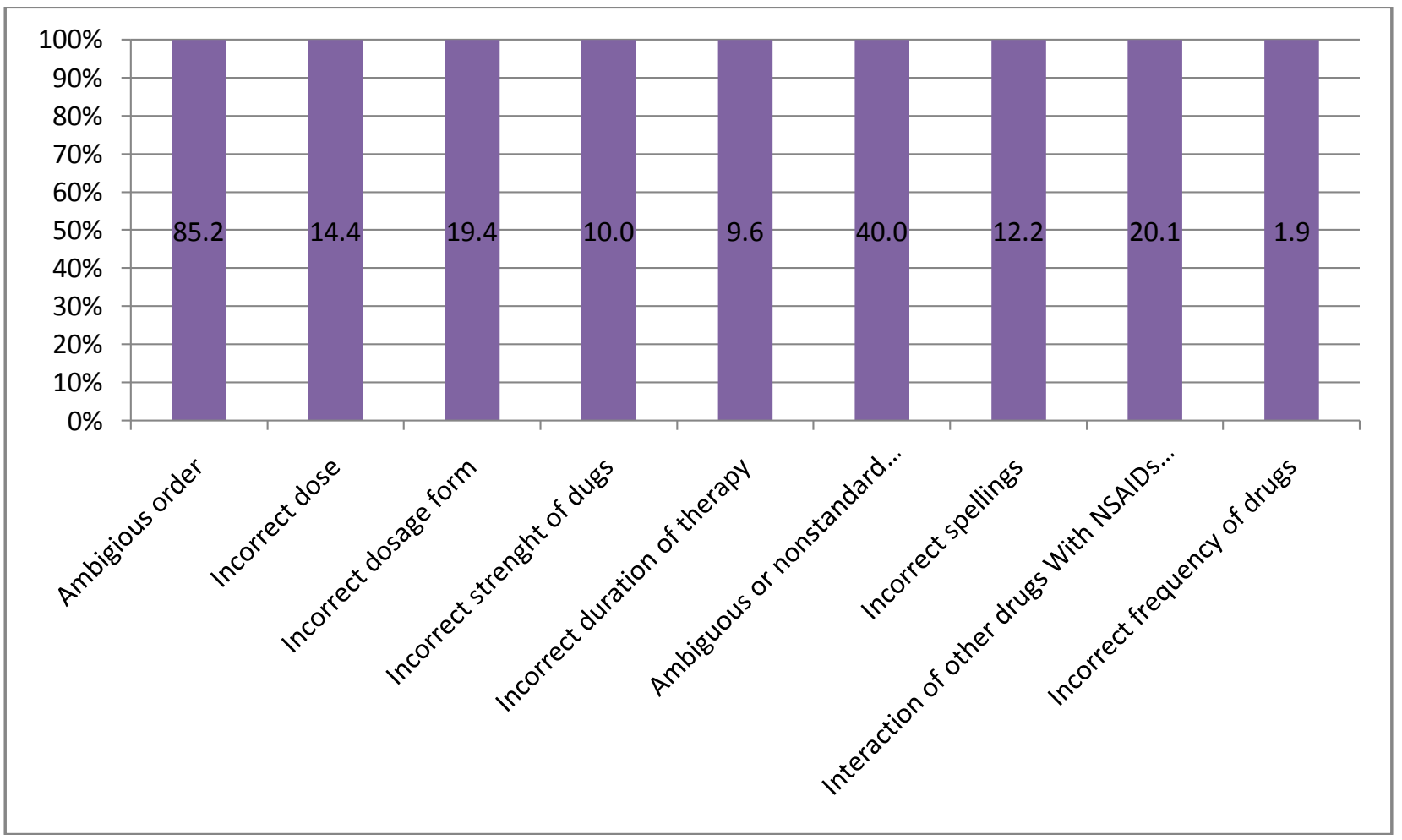

Fig. 4: Graphic presentation of commission errors in prescriptions 
Table 1: Patient related information in prescriptions

\begin{tabular}{|l|c|c|}
\hline Parameters related with Patient information & Mentioned N (\%) & Not MentionedN $(\%)$ \\
\hline Name & $446(97.4)$ & $381(2.6)$ \\
\hline weight & $77(16.8)$ & $385(84.1)$ \\
\hline Diagnosis & $73(15.9)$ & $240(52.4)$ \\
\hline Sex & $218(47.6)$ & $275(60.0)$ \\
\hline Age & $183(40.0)$ & \\
\hline
\end{tabular}

Table 2: Prescriber related information in prescriptions

\begin{tabular}{|l|c|c|}
\hline Parameters related with Prescriber information & Mentioned N (\%) & Not MentionedN (\%) \\
\hline Name & $211(46.1)$ & $247(53.9)$ \\
\hline Address & $301(65.7)$ & $157(34.3)$ \\
\hline Telephone number & $269(58.7)$ & $489(41.3)$ \\
\hline Registration number & $51(11.1)$ & $407(88.9)$ \\
\hline Qualification & $59(12.9)$ & $399(87.1)$ \\
\hline Signature & $411(89.7)$ & $47(10.3)$ \\
\hline
\end{tabular}

Table 3: Drug related information in prescriptions

\begin{tabular}{|l|c|c|}
\hline Parameters related withDrug information & MentionedN (\%) & Not MentionedN (\%) \\
\hline Brand name & $411(89.7)$ & $47(10.3)$ \\
\hline $\begin{array}{l}\text { Strength, when more than one strengths of } \\
\text { same are available in market }\end{array}$ & $188(41)$ & $270(59)$ \\
\hline Direction of use & $99(21.6)$ & $359(78.4)$ \\
\hline Dose & $344(75.1)$ & $114(24.9)$ \\
\hline Dosage form & $397(86.7)$ & $61(13.3)$ \\
\hline Duration of therapy & $79(17.2)$ & $379(82.8)$ \\
\hline Frequency of drugs & $341(74.5)$ & $117(25.5)$ \\
\hline
\end{tabular}

Table 4: Information of commission errors in prescriptions

\begin{tabular}{|l|c|}
\hline Commission error type & Number Of Prescriptions (\%) \\
\hline Ambiguous order & $390(85.2)$ \\
\hline Incorrect dose & $66(14.4)$ \\
\hline Incorrect dosage form & $89(19.4)$ \\
\hline Incorrect strength & $46(10.0)$ \\
\hline Incorrect duration of therapy & $44(9.6)$ \\
\hline Ambiguous or nonstandard abbreviations of drug names & $183(40.0)$ \\
\hline Incorrect spellings & $56(12.2)$ \\
\hline Interaction of other drugs With NSAIDs in prescriptions & $92(20.1)$ \\
\hline Incorrect frequency & $9(1.9)$ \\
\hline
\end{tabular}

\section{Conclusion:-}

It is summarized that huge number of omission errors and commission errors are occurring during the writing of prescriptions and these errors are not only seriously affecting the therapeutic outcomes of treatment but also raising the needless expenditures and utmost of the errors are linked to not mentioning essential information or mentioning inappropriate information of the patient, prescriber and drug. These errors in prescriptions can easily cause incorrect dispensing, incorrect administration of medicine, drug abuse and drug interactions. It is proposed that implementation of computerized physician order entry (CPOE) and continuous educational and training programs of prescriptions writing guidelines/standards for prescribers can meaningfully reduce the extent of prescribing errors. Most of the hospitals have computerized physician order entry systems and they found that a significant proportion of errors were eliminated. The registered pharmacists can also play an important role in minimizing and avoiding these errors by assessing the prescriptions. 


\section{Acknowledgement:-}

We are grateful of all supporters of this study for their kind help in the gathering of prescriptions throughout the study.

\section{References:-}

1. Bates D, Boyle D, Vander Villet M, Schneider J, Leape L. (1995). Relationship between medication errors and adverse drug events. J Gen Intern Med. 10:199-205.

2. Scott, S.A.The Prescription. (2005): In Remington: The Science and practice of pharmacy, $21^{\text {st }}$ ed. Philadelphia: Lipponcot.Williams \& Wilkins: p.1823-25.

3. De Vries TP., Henning, R.H., Hogerzeil, H.V.and Fresle DA.(1995): Guide to Good Prescribing. In: World Health Organization, editor.A Practical Manual,(WHO/DAP/94.11). Geneva: p. 52-55.

4. Cooper J.W.Cooper and Gunn's dispensing for pharmaceutical students (1975).Pitman Medical.

5. NCCMERP (2012). The National Coordinating Council for Medication Error Reporting and Preventing.Available from: www.nccmerp.org.

6. Manasse HR Jr. (1989): Medication use in an imperfect world:drugmisadventuring as an issue of public policy. Am J Hosp Pharm., 46:929-44.

7. Dean, B., Barber, N., andSchachter, M. (2000): What is a prescribing error? Qual Health Care., 9:232-37.

8. Ansari, M., and Neupane D.(2009): Study on determination of errors in prescription writing: A semi-electronic perspective. Kathmandu Univ Med J., 7:238-41.

9. Mugoyela, V and Mwita S. (2008): Extent of Occurrence of Prescribing Errors in a Private Tertiary-Care Hospital in Dar es Salaam. Tanzania Medical Journal. 23:20-22.

10. Ekedahl A. (2010). Problem prescriptions in Sweden necessitating contact with the prescriber before dispensing. Res Social Adm Pharm. 6(3):174-84.

11. Lesar, TS. (2002): Prescribing errors involving medication dosage forms. J Gen Intern Med., 17:579-87.

12. Gosney, M.R. andTallis. (1984): Prescription of contraindicated and interacting drugs in elderly patients admitted to hospital. The Lancet., 324:564-67.

13. Ridley, S.A., Booth, S.A and Thompson, C.M.(2004): The Intensive Care Society's Working Group on Adverse Incidents. Prescription errors in UK critical care units. Anesthesia., 59:1193-1200.

14. Bemt, V.D., Patricia, M.L.A., Postma, M.J., Van,R.E.N., RoelFijn,C.M.C.andJacobus RBJ.(2002): Costbenefit analysis of the detection of prescribing errors by hospital pharmacy staff. Drug Safety.,25:135-43.

15. McGettigan,P.andHenry,D. (20013): Use of non-steroidal anti-inflammatory drugs that elevate cardiovascular risk: an examination of sales and essential medicines lists in low-, middle-, and high-income countries. PLoS medicine., 10:1001388.

16. Rodríguez, L.A.G. and Hernández-Díaz S. (2001): Relative risk of upper gastrointestinal complications among users of acetaminophen and nonsteroidal anti-inflammatory drugs. Epidemiology., 12: 570-76.

17. Thomas, J., Straus, W.L. and Bloom, B.S.(2002): Over-the-counter nonsteroidal anti-inflammatory drugs and risk of gastrointestinal symptoms. Am. J. Gastroenterol.,97: 2215-19

18. Lacy, C.F., A.L., Goldman, MP and Lance LL.(2010):Drug Information Hand book, 19th Edition. Lexi-comp, Inc. Hudson, ohio.

19. British National Formulary. (March 2010): edition 59th British Medical Association and Royal Pharmaceutical Society of Great Britain, London.

20. DRUG-REAX (Micromedex). (2005): Thomson Micromedex. Greenwood Village, Colorado, USA: DRUGREAX (Micromedex).

21. Shumaila, S., Muhammad, A., Omer, M., Muhammad, A.G., Hina, S., Sumbul, S. and Abdullah D. (2014): A retrospective study of NSAIDs containing medication orders for evaluating prescribing errors in outpatient clinical settings of a metropolitan city. Journal of Pharmacy and Biological Sciences., 9:141-46.

22. Shagufta, N., Muhammad, H.S., Najia, R., Wajiha, I., Sadia,S and Rehana B. (2015):Prescription Writing Practices and errors in prescriptions containing cardiovascular drugs specially ACE inhibitors in Karachi, Pakistan. Asian J Pharm Clin Res., 8:53-5.

23. Ghoto, M.A., Abdullah, D., Muhammad, A., Imran, S. and Abbas, A. (2013): Identification of errors in antibiotics' prescriptions and prescription writing trends in areas of Hyderabad Sindh, Pakistan. Afr J Pharm Pharmacol., 7:1009-14.

24. Phalke,V.D., Phalke, D.B., Syed, M.M., Mishra, A., Sikchi,S.andKalakoti P.(2011): Prescription writing practices in a rural tertiary care hospital in Western Maharashtra, India. Australas Med J., 4:4-8. 
25. Irshaid, Y.M., Al,Homrany., M, Hamdi A.A., Adjepon-Yamoah, KK and Mahfouz AA.(2005): Compliance with good practice in prescription writing at outpatient clinics in Saudi Arabia. East Mediterr Health J.,11:9228.

26. Banks, Alpha T., Zimmerman,HJ.,Ishak,K.G.and Harter JG. (1995): Diclofenac-associated hepatotoxicity: Analysis of 180 cases reported to the food and drug administration as adverse reactions. Hepatology., 22:82027.

27. Stubbs, Haw JC.andTaylor D. (2006): Prescription errors in psychiatry-a multi-centre study. Journal of Psychopharmacology.,553-61. 